tungshof muß auf das umgebende Plasma einen Zug ausüben; sind nun die Teilchen des Plasmas nicht ungehindert verschiebbar, d. h., können sie sich unter der Zugwirkung nicht sofort wieder so umordnen, wio das bei den Teilchen einer homagenen dünnflüssigen Masse der Fall wäre, so entsteht um die Verdichtungszone eine Strahlenfigur. Am leichtesten und klarsten läBt sich die Entstehung solcher Zugstrahlen theoretisch für ein schaumig-wabiges Substrat klarmachen, ja, man kann sagen, daß gerade diese Erklärung der Strahlenbildung später durch Rhumbler zu einem der hübschesten Kapitel der Wabenlehre wurde. - Die Grundlage der theoretischen Ableitungen bildeten für Bütschli rein physikalische Beobachtungen an toten kolloiden Medien: auch in dicker, erstarrender Gelatine konnte er künstlich um sich verkleinernde Zentren (z. B. sich zusammenziehende Luftbläschen) die schönsten Strahlungen und zwischen zwei nahe beieinanderliegenden Zentren vollständige ,karyokinetische Figuren" entstehen lassen, deren "Ahnlichkeit mit den Zellgebilden in allen Einzelheiten ganz verblüffend wirkt - und das alles einfach durch die physikalischen Faktoren, welche wir oben theoretisch erörterten. Weil sich nun aber gerade diese Faktoren auch alle auf die Verhältnisse in der lebenden Zelle übertragen lassen, müssen uns Bütschlis künstlicho Astrosphären als eines der ersten Musterbeispiele eines, physikalischen Modells" einer Lebenserscheinung erscheinen, eines Modelles, das selbsttätig durch rein physikalische Kräfte den Febenden Zellgebilden Analoges entstehen läßt. Solche Modelle sehen wir heute als die wichtigsten Bausteine der jungen Wissenschaft der ,synthetischen Biologie" an.

\title{
Otto Bütschli als Kolloidchemiker.
}

Von H. Freundlich, Berlin-Dahlem.

Es konnte einem aufmerksamen Beobachter der Lebensvorgänge nicht entgehen, daß bei diesen Gallerten, amorphe Flocken, trübe, zähe, schäumende Flüssigkeiten, kurzum Gebilde, wie sie in der Kolloidchemie erforscht werden, eine bedeutsame Rolle spielen, eine weit größere, als etwa gut gewachsene Kristalle oder klare, leichtflüssige, nicht schäumende Lösungen. Man mag in diesem Zusammenhang an eine Stelle in ,Wahrheit und Dichtung" denken: Bei seinen alchimistischen Versuchen bereitete Goethe aus den Mainkieseln Wasserglaslösungen und, die schönste mineralische Flüssigkeit, die mir einige Male zu meiner größeren Verwunderung in Form einer animalischen Gallerte erschienen war, ließ doch immer ein Pulver fallen, das ich für den feinsten Kieselstaub ansprechen muBte, der aber keineswegs irgend etwas Produktives in seiner Natur spüren lie $\hat{B}$, woran man hätte hoffen können, die jungfräuliche Erde in den Mutterzustand übergehen zu sehen." Als dann Graham den Begriff der Kolloide formte, war er sich durchaus darüber klar, wie wichtig sie für die Erklärung der. Lebensvorgänge sein würden; ja die t'berzeugung, daß die ,kolloiden Stoffe" bevorzugt in der Organismenwelt enthalten sind, verleitete ihn vielleicht mit daza, so schroff von zwei Stoffgruppen zu sprechen, die, wie verschiedene Welten der Materie erscheinen" - eine Unterscheidung, die sich bekanntlich nicht bewährt hat.

Otto Bütschli war wohl der erste Forscher, dem das Aussprechen einer solchen oberflächlichen Ahnlichkeit nicht genügte. Mit aller Klarheit sah er ein, daß das, was sich physikalisch-chemisch an den Lebensvorgängen erklären läBt, auch in dieser Weise aufgeklärt werden muB, ehe die Frage nach nicht physikalisch-chemischen "Einflüssen berechtigt ist. Ihm, dem mineralogisch Geschulten, fiel
- wie es nur verständlich ist - die kolloide Beschaffenheit des Protoplasmas, der Membrane, überhaupt fast all der Elemente, die einen Organismus zusammensetzen, besonders auf; ihre Wichtigkeit für die Lebensvorgänge war für ihn so überzeugend, daß er die Arbeit einer langen Reihe seiner besten Mannesjahre ihrer Erforschung widmete. Er stieB ron dem Standpunkt aus, von dem er als Biologe in die Kolloidchemie eindrang, sozusagen auf die unangreifbarste, am stärkstea verschanzte Flanke der zu erobernden Festung: Denn die Systeme, mit denen er sich notwendig am eingehendsten befassen muBte, die Gele überhaupt, als Membrane, als Fasern, als Sphärokristalle, gehören heute noch zu den Gebilden, deren Eigenschaften und Verhalten vielfach nur ganz unvollkommen erklärt werden könneil trotz der ausgedehnten kolloidchemischen Arbeit der letzten beiden. Jahrzehnte. $E_{s}$ ist deshalb nicht erstaunlich, daß es ihm nicht immer gelang, die endgültige Lösung der von ihm aufgeworfenen Fragen zu finden.

Die Mehrzahl von Bütschlis kolloidchemischen Arbeiten befaBt sich mit dem Bau der Gallerten oder Gele, und zwar bemühte er sich, unmittelbar durch mikroskopische Untersuchungen in ihr Wesen einzudringen. Er war sich dabei durchaus bewuBt, daB die mikroskopischen Beobachtungen nicht leicht zu deuten sind, wenn die Strukturen, mit denen man es zu tun hat, von der Größenordnung der Wellenlänge des sichtbaren Lichtes nicht weit entfernt sind, und er suchte dieser Unsicherheit in verschiedener Weise zu begegnen: Er überzeugte sich einmal von dem Aussehen und Verhalten der mikroskopischen Bilder möglichst kleiner Tröpfchen und Luftbläschen. Dann änderte er die Eedingungen seiner Versuche in jeder denkbaren Richtung ab und dehnte sie über eine mög- 
lichst'große Zahl von Gelen verschiedener Art aus.

Zwei Gedanken sind es, auf die ihn seine Beobachtungen immer wieder von neuem führten, und die er unermüdlich weiter verfolgte; einmal, daB die Gele zweiphasige Gebilde sind utd dann, daß sie einen schaumig-wabigen $\mathrm{Bau}$ haben, also aus kleinen Flüssigkeitströpfchen bestehen, die von fëinen amorph-festen Wänden umhüllt sind. An der Zweiphasigkeit der Gele werden heute nur noch wenige zweifeln, mag man auch formal manche Vorgänge bei der Quellung vom Stand punkte der festen Lösungen, also eines einphasigen Gebildes, aus erfolgreich darstellen können. Weniger wahrscheinlich ist es, daß der schaumig. wabige Bau so allgemein zutrifft, wie es Bütschli glaubte. Er hielt ihn keineswegs für den allein möglichen oder notwendigen, sondern erörterte daneben auch eine "globulitisch-wabige" Struktur, d. h. eine Struktur, bei der zunächst gesonderte Globulite vorhanden sind; diese können $\mathrm{zu}$ Schichten zusammentreten, in denen sie, anfangs getrennt, erst im Laufe der Zeit verschmelzen. Aber er neigt doch der Meinung zu, daß überwiegend, auch z. B. bei den Gelen der Kieselsäure, der Gelatine u. a., eine schaumig-wabige Struktur vorhanden ist, also zusammenhängende amorphfeste Schichten, die die flüssigen Tröpfchen umkleiden; anders ausgedrückt: $\mathrm{Er}$ betrachtet den amorph-festen Stoff als Dispersionsmittel, die Flüssigkeit als disperse Phase, während bei der globulitisch-wabigen Struktur zunächst die Flüssigkeit Dispersionsmittel, die amorph-festen Teilchen die disperse Phase sind.

Nun hatte Bütschli diesen Bau bei den genannten Gelen nicht unmittelbar an dea erstarrten Gelen beobachtet, sondern nur unter besonderen Versuchsbedingungen; bei den Gelatinegelen z. B., wenn sie vorsichtig mit Chromsäure gegerbt waren, bei den Kieselsäuregelen vorübergehend im Gebiet des sogenannten Umschlagens, d. h. während das Gel beim Eintrocknen sich mit Luftblasen füllte, oder die Luft beim Tränken mit Flüssigkeit entwich. Er glaubte aber schließen zu dürfen, daß durch die besonderen Versuchsbedingungen der schaumig-wabige Bau nur besser sichtbar gemacht würde. Neuere Untersuchungen von Zsigmondy und Bachmann lassen es fraglich erscheinen, ob dieser Schluß berechtigt ist. Sie fanden ultramikroskopisch eine feinere Körnung, als sie Bütschli mikroskopisch beobachtet hatte, und zwar benutzten sie Gele, die sie nicht weiter. vorbehandelt hatten, sondern die einfach aus den Solen erstarrt waren. 'Diese feinere Körnung war nicht schaumig-wabig, sondern man hatte ultramikroskopisch feine Globulite, die durch ultramikroskopisch feine Flüssigkeitshäute getrennt waren. Die Flüssigkeit war also Dispersionsmittel, der feste Stoff disperse Phase. Es ist demnach die Nägelische Micellartheorie, die für diese Gele bestätigt wird. Die von Bütschli gefundene mikroskopische Körnung ist dann anders zu erklären: Bei den Gelatinegelen handelt es sich um gröbere, durch die Gerbung erzeugte Gebilde, wohl von globulitisch-wabiger Struktur; bei den Kieselsäuregelen sieht man wahrscheinlich den Schaum feinster Gasbläschen, die in dem noch zum Teil mit Flüssigkeit erfüllten Gelraum verteilt sind.

Kann man danach aus Bütschlis Beobachtungen auch nicht auf den Bau der Gele ohne weiteres schließen, so läBt sich doch eine Fülle anderer Folgerungen ziehen, und sie werden um so fruchtbringender sein, je mehr man sich mit der feineren Struktur der Gele befaßt, ein Gebiet, dessen Erforschung man nur erst begonnen hat. So sind die kleinsten Teilchen eines Gels, die Micellen, sicher nicht unabhängig voneinander, sondern in bestimmter Weise geordnet und zum Teil verschmolzen. Dies äußert sich z. B. darin, $\mathrm{da} B$ Gasblasen, die man in einem Gelatinegel erzeugt hat, nicht immer kugelförmig sind, sondern sehr oft linsenförmig: Mit dem Spannungszanstand, der sich hierin äußert, hängen wohl auch die von Bütschli beschriebenen Strukturveränderungen in der gegerbten Gelatine zusammen, die auftreten, wean eingelagerte Luftblasen ihre Größe verändern, und die er wegen ihrer Ahnlichkeit mit bei der Karyokinese auftretenden Formen eingehend verfolgt hat. Nicht minder die von ihm untersuchten merkwürdigen, regelmäßigen Sprungsysteme, wie sie in Harzschichten entstehen, die eine quellendo Gelatineschicht überziehen. Seiner Aufmerksamkeit ist es ferner nicht entgangen, daß in einem Gel durch einseitigen $\mathrm{Zug}$ die Körnung in regelmäßiger Weise verschoben wird. Wie sich neuerdings herausgestellt hat, kommt es dabei sehr auf die Gestalt der Micellen an; sind sie z. B. längestreckt, so. lagern sie sich mit ihrer Längsachse in die Zugrichtung, und es ist wahrscheinlich, daB die Eigenschaften der natürliehen wie künstlichen Fasern stark durch diese Ümstände mitbedingt werden.

Schließlich ist nicht zu vergessen, $d a B$ viele der von Bütschli untersuchten verwickelteren Gebilde, die sicher einen schaumig-wabigen Bau haben, wie z. B. die Emulsionen feinster Oltröpfchen in einer Gelatinegallerte, wahrscheinlich den natürlich vorkommenden Gebilden weit mehr ähneln, als es verhältnismäBig so einfacho Gebilde tun, wie es die Gele der Gelatine oder der Kieselsäure sind. Für die weitere Aufklärung der Lebenserscheinungen hat es gewiB etwas MiBliches, wenn man immer wieder betont, wie verwickelt sie sind, zumal da oft die Forscher recht zu behalten scheinen, die die Einfachheit der $\mathrm{Na}$ tur hervorheben. Die Wahrheit liegt wohl in der Mitte: Einfach erscheinen die in der Natur, insbesondere auch bei den Lebenserscheinungen stattfindenden Vorgänge, wenn es gelingt, irgend zwei Einflüsse in ihrem gesetzmäßigen Zusammenhang möglichst frei von weiteren Umständen herauszuschälen. Sie sind aber gewiß nicht in 
dem Sinne einfach, daß nur eine kleine Zahl von Stoffen oder Phasen beteiligt ist. Hier kann man vermutlich die Mannigfaltigkeit gar nicht grob genug einsetzen, und Protoplasma, Membrane und dergl. sind nicht einfach zweiphasige Gebilde, wie sie die Kolloidchemie bisher wesent. lich erforscht hat, sondern enthalten eine viel größere Zahl von Phasen, die beim Protoplasma wohl schaumartig angeordnet sind.

Noch eine kolloidchemische Untersuchung Bütschlis sei besonders hervorgehoben. Er wies darauf hin, da $B$, wenn sich eine etwa quellbare Lamelle auf der einen Fläche anders ausdehnt als auf der anderen, Einstülpungen und Faltungen statthaben können, die auffallend den Formen gleichen, wie sie z. B. eine lebende Zellplatte bei der Gastrula-Invagination erfährt. Spek hat von diesem Gedanken aus Lamellen hergestellt, deren eine Seite aus Agar, deren andere aus einem aus
Gelatine + Agar bereiteten Gel bestand, und hat gezeigt, wie man auf Grund der Quellungsverschiedenheit der beiden Seiten Modelle formen kann, deren Faltungen selbst Einzelheiten des genannten biologischen Vorganges darzustellen erlauben. Nicht nur derartige Modelle wird man in $\mathrm{Zu}$ kunft in immer weiterem Umfang $\mathrm{zu}$ gestalten lernen; man wird so wohl auch dazu gelangen; die Quellungsmotoren und Kapillarelektromotoren zu bauen, die man bisher im Laboratorium und in der Praxis kaum kennt, während sie die Natur vor allen anderen verwendet.

Die Kolloidchemie darf hoffen; mehr und mehr als eine der Grundlagen anerkannt zu werden, auf der sich die Lehre von den Lebensvorgängen aufbaut, und Bütschli wird stets als einer der ersten genannt werden, der für diese Auffassung mit seinen Worten und seiner Arbeit bahnbrechend und erfolgreich eingetreten ist.

\title{
Otto Bütschlis Verhältnis zor Kristallographie und Mineralogie.
}

\author{
Von V. Goldschmidt, Heidelberg.
}

L's ist immer von Wert, wenn ein Forscher sich nicht auf sein engstes Gebiet beschränkt, sondern auch in anderen Gebieten des Wissens sich auskennt. Leibnit $z$ war Jurist, Philosoph und Mathematiker, Helmholtz war Physiker, Mathematiker und Physiolog, der geistreiche Mineralog Franz $v$. Kobell war nebenbei Jäger und Volksdichter. Fin Gebiet wirkt befruchtend auf das andere. Nir die kleinen Flickschuster bleiben am besten bei ihrem Leisten.

Der Zoologe Bütschli war zterst Mineralog. Eine Zeitlang war er Assistent für Mineralogie an der Technischen. Hochschule in Karlsruhe. Diese eingehende Beschäftigung mit dem Anorganischen, mit der Mineralchemie und der anorganischen Mikroskopie hat vielen seiner Arbeiten Inhalt und Richtung gegeben. Bis zum Ende seines Lebens beschäftigte ihn, den Zoologen, die Struktur der Kristalle und er studierte die Beziehungen zwischen den organischen und den anorganischen Strukturen, so besonders beobachtete er die Struktur der anonganischen $\mathrm{Ge}$ bilde im Tierkörper, die Schalen der Mollusken, Krebse und Echiniden, die Gerüste der Rhizopoden und Foraminiferen, die Kalk- und Kieselnadeln der Schwämme. Die Mineralchemie führte zu seinen Untersuchungen über organische Kalkgebilde nebst Bemerkungen über organische Kieselgebilde. Sie brachten auch eine reizende kleine Arbeit von Bütschli, ,Uber die Loslichkeit des Schwefels in Wasser und Glycerin'1). Dieselbe aüfte den meisten Zoologen unbekannt geblieben sein, auch bei den Kristallographen hat sie wenig Beachtung gefunden Es scheint daher gut, einen Aüszug a ưs derselben hier wieder

1) Zéritschr. Krist. $1899 ; 31,277-279$. zugeben und die Resultate zu diskutieren. Ich selbst habe mich vorher mit ähnlichen Versuchen beschäftigt, es waren meine Versuche Bütschli bekannt. Ja ich verdanke seiner mikrophotographischen Aufnahme die Bilder in meiner diesbezüglichen Publikation (1897)2). Die bei Bütschlis Versuchen gebildeten Schwefelkriställchen hat W. Salomon untersucht und in ihnen die sog. 3. Modifikation des Schwefels nachgewiesen.

Kristallisation aus der Unterschmelze. Andere nennen das tberschmelze. Es ist ein Flüssigbleiben unter dem Schmelzpunkt. Zunächst möge aus meiner Publikation') ein Passus wiedergegeben werden. Die dort beobachtete Substanz heißt Furfuraldoxim. Wir lesen (S. 170):

„Verialten bei der Entschmelzung. Die Substanz schmilzt leicht zu farblosen Tropfen. Bei Abkühlung auf Zimmertemperatur erstarrt sie nicht von selbst, wohl aber in Berührung mit einer Nadel oder mit einem Fristall der eigenen Substanz.

Wir schmelzen auf einem Objektträger eine Anzahl Kriställchen ein, die nebeneinander getrennte Tröpfchen bilden, lassen sie abkühlen, bringen sie unter das Mikraskop, mit schwacher, etwa 60 facher VergröBering und wählen zwei. Tropten (F und $E$ ) aus, die zufällig an einer Stelle nahe aneinanderliegen. Von diesen entschmelzen wir den einen $(K)$ durch Berühren mit einem Kristall seiner Substanz, von dem $F^{\prime}$ : entgegengesetzten Ende aus. Es schieben von der Berührungsstelle aus Kristallnadeln fächerförmig an und wachsen rasch weiter bis zur Grenze des Tropfens.

Beobachtet man nun beide Tropfen, den flüssigen (F) und den aúskristallisierten $(K)$ in dér Gegend ihrer Annäherung unter dem Mikroskop, so zeigt sies folgendes $\mathrm{Zuerst}$ hat $K$-das Aussehen fig. 1.

2) Uber einen interessanten Fall der kristallinen Entschmelzung. Zeitschr. Krist. $1897,28,169-173 \mathrm{u}$ Taf, 3, Fig. $3-\bar{z}$. 